\title{
La ruse à la frontière des genres : regards croisés sur les farces de Molière et les contes de La Fontaine
}

\author{
Jean Leclerc ${ }^{1}$ \\ Université Western Ontario
}

Durant l'Ancien Régime, une parenté forte unit les genres de la farce et du conte grivois ${ }^{2}$, que ce soit l'intérêt pour les thèmes tirés du quotidien et de la vie conjugale, les ressorts du comique

\footnotetext{
${ }^{1}$ Cet article est une version remaniée d'une communication présentée lors de la Kentucky Foreign Language Conference en avril 2011, dans la séance « Erreur, ruse et mensonge au XVIIe siècle en France» organisée par Gilles Declercq. La recherche nécessaire a été accomplie grâce à une subvention du CRSH.

${ }^{2}$ La nomenclature entourant la narration brève est des plus fluctuantes. Ménard intitule son étude Les Fabliaux : contes à rire du moyen âge (1983), tandis que La Fontaine parle indistinctement de contes ou nouvelles dans ses titres de recueil.
} 
comme le bas corporel ou le style familier, si bien qu'ils partagent plusieurs frontières communes et que ces frontières se chevauchent ou se brouillent même parfois. La farce prolonge sur la scène la matière et la manière du conte. Certes, la bienséance ne permet pas qu'on représente sur scène les choses de l'amour alors qu'on peut se permettre de les narrer plus allusivement dans les contes, où la grivoiserie pouvait être poussée plus avant. Mais si l'on admet que le mariage qui termine toute comédie n'est que l'étape préliminaire et obligée à l'accomplissement de l'acte sexuel, qui est sa conséquence immédiate, on pourrait croire que cette cérémonie n'est représentée que pour les formes, sorte d'euphémisme en regard des exploits amoureux décrits dans les contes. La farce serait alors à la représentation scénique ce que le conte grivois, et son ancêtre le fabliau, est à la narration, que ce soit en vers ou en prose. Il s'agirait donc de deux genres miroir, qui posséderaient une frontière commune qui s'articulerait autour des éléments triviaux du style et de la thématique, mais qui pourrait aussi s'articuler autour d'un élément de l'intrigue, dans la mesure où ces deux genres utilisent la ruse comme procédé narratif.

Le concept de la ruse s'avère un outil efficace afin de mieux percevoir les similarités et les différences entre le conte plaisant et le théâtre comique ${ }^{3}$, non seulement parce que les deux en font un usage presque systématique, mais aussi parce qu'ils l'utilisent souvent aux mêmes fins, c'est-à-dire pour faire avancer l'intrigue et susciter un rire de connivence avec le destinataire. La ruse constitue un ressort privilégié du comique

\footnotetext{
3 On verra comment la séparation entre la farce et les comédies plus régulières ne tient pas dans le cas de Molière. L'ampleur du corpus étudié Rey-Flaud (1996) prouve bien que la longueur n'est pas le seul critère de distinction entre ces deux genres.
} 
narratif et scénique, de la Farce de maître Pathelin au Mariage de Figaro, en passant par Larivey, Scarron, Molière et Marivaux ${ }^{4}$. De même, la réutilisation des sujets similaires fait en sorte que la ruse est repérable autant dans les Cent nouvelles nouvelles que chez La Fontaine, en passant par l'Italie avec Boccace, le Pogge et Straparole, jusqu'en France avec Bonaventure des Périers, Noël du Fail et Marguerite de Navarre ${ }^{5}$. On peut prendre ce terme au sens large que lui confèrent les dictionnaires depuis le XVIIe siècle « Finesse, artifice, moyens subtils dont on se sert pour tromper » (Dictionnaire de l'Académie française) - ou préférer les indications similaires du Trésor de la langue française: "Procédé habile dont on use pour tromper» (Imbs). Toutes ces définitions font voir une complémentarité entre un procédé mis en œuvre et une finalité associée à une intentionnalité. Les auteurs de l'époque étaient bien au fait des potentialités comiques de la ruse. Dès la fin du XVI ${ }^{\text {e }}$ siècle, Laudun d'Aigaliers écrivait: la "matière [de la comédie] est toute ruse et tromperie » (cité par Rey-Flaud, p. 19). Corneille lui-même s'en servait pour distinguer le comique du tragique dans l'épître dédicatoire de La Suivante (1637): «Les fourbes et les ruses sont principalement du jeu de la comédie, les passions n'y entrent que par accident. » (cité par Sternberg, p. 37) Que la ruse soit perçue par les critiques comme un thème, un procédé du comique ou une composante de l'intrigue, elle occupe une place centrale au sein de cette littérature aux côtés de la satire sociale, de la peinture des caractères, du quiproquo et du malentendu.

\footnotetext{
${ }^{4}$ D'une assez large bibliographie sur la farce, on retiendra les incontournables travaux de Bowen (1964) et de Lewicka (1974). Sur l'histoire et la poétique de la comédie, voir Voltz (1964), Gilot et Serroy (1997) et Sternberg (1999). ${ }^{5}$ Sur l'histoire du conte, en France comme en Italie, voir Godenne (1995) et Souiller (2004).
} 
La continuité de la farce et du conte plaisant ne se trouve nulle part illustrée de façon plus claire que dans les œuvres de Molière et de La Fontaine. L'étude conjointe des pièces de l'un et des contes de l'autre en regard de la ruse n'a pas encore été entreprise ${ }^{6}$, bien que des ouvrages monographiques proposent au passage des analyses pertinentes de ce ressort du comique. Catherine Grisé a publié récemment une étude sur la tromperie et l'illusion dans les fables et les contes de La Fontaine; plusieurs critiques ont parlé de la ruse, de la «bourla» ou de la fourberie chez Molière, notamment Patrick Dandrey dans ses travaux sur la médecine (1998), Antony McKenna sur le libertinage (2005), Jean de Guardia sur le comique de répétition (2007), sans oublier l'ouvrage déjà cité de Bernadette Rey-Flaud sur la farce (1996). Outre les affinités des deux genres qui ont fait une partie de leur succès, d'autres aspects historiques et culturels justifient un rapprochement entre Molière et La Fontaine. Fortement soupçonnés d'épicurisme, ces deux auteurs ont voulu divertir un public qui ne cachait pas son penchant pour l'hédonisme, que ce soit l'entourage royal dans le cas de Molière ou la cour du surintendant Fouquet pour La Fontaine, du moins à ses débuts. Tous deux ont rencontré plusieurs détracteurs : quelques pièces de Molière ont été interdites, voire réécrites, afin d'être représentées, tandis que les Nouveaux contes de 1674 ont fait l'objet d'une interdiction et d'une saisie par le lieutenant général de police La Reynie? D’ailleurs, son élection à l'Académie sera fortement compromise par la rédaction de ces contes licencieux.

\footnotetext{
${ }^{6}$ Parmi les rares ouvrages sur l'écriture de la ruse sous l'Ancien Régime, mentionnons Grodek (2000).

${ }^{7}$ Collinet (1970), p. 333. Voir aussi sa notice sur les Contes dans son édition des CEuvres complètes (1991), t. I, p. 1333. Toutes les indications renvoient à cette édition.
} 
Une analyse de la ruse chez ces deux auteurs peut faire voir l'appartenance à une longue tradition littéraire, mais peut permettre en retour d'interroger les sensibilités propres à la décennie 1660, la proximité des deux auteurs sur le plan idéologique et moral, et de mieux illustrer la frontière entre les genres de la farce et du conte grivois. Pour ce faire, trois axes apparaissent particulièrement profitables : d'abord à travers un questionnement sur les a priori éthiques ou juridiques entourant la pratique de la ruse et ses conditions de réception auprès du public; ensuite par l'interrogation de la topique de la ruse, qui s'attardera à ses principaux ressorts; enfin en posant un regard sur les abus de logique propres au discours de la ruse, c'est-à-dire tous ces lieux où la raison est prise en défaut afin de faire rire et de faire réfléchir, faisant de cette littérature un lieu d'apprentissage des mœurs et des procédés d'argumentation.

\section{Les conventions éthiques et juridiques}

Pour que fonctionne le rire fondé sur une ruse, il faut préserver ce que Bergson appelait l'«anesthésie momentanée du cœur» (Le rire, cité par Sternberg, p. 37 », sans quoi les réflexes de sympathie et de pitié propres à l'être humain feraient percevoir une injustice et amèneraient le public à partager les doléances du personnage trompé. Le contexte comique appelle une entente tacite entre l'auteur et le public, fondée d'une part sur la certitude qu'aucun personnage ne subira de graves dommages, comme la mort, les blessures qui handicapent, la perte de l'honneur ou des biens, et, d'autre part, sur l'établissement d'une fin visiblement légitime qui justifiera l'utilisation de moyens parfois jugés immoraux. La scène 
ou le livre crée en outre une distance esthétique permettant de mettre en évidence l'aspect fictionnel du spectacle ou de la narration, rendant inoffensives des procédures qui seraient outrageantes dans la réalité ${ }^{8}$. La ruse comique opère alors sur un terrain moral différent de celui sur lequel se placent les moralistes et les théologiens, qui sont unanimes à condamner la tromperie, le manque de loyauté familiale, le mensonge ou le déguisement. C'est ce qui explique aussi pourquoi la ruse conviendrait mal à un personnage comme Rodrigue: Le Cid relève d'une morale généreuse où l'honnêteté et la magnanimité forment les principales qualités du héros (voir Bénichou, p. 15-67).

Pour ne pas être entièrement condamnables, la comédie et le conte atténuent la gravité morale de la ruse par une justification contextuelle propre à l'intrigue. Elle est plus souvent un mécanisme de légitime défense que d'agression, une réaction face à une situation abusive et indésirable. La plupart du temps, la fin, toujours bonne en elle-même, justifiera les moyens pris pour y arriver, atténuera les contrecoups moraux qui font du mensonge et de la tromperie des fautes graves. Par exemple, l'amour entre les jeunes premiers sera toujours une cause plus forte que l'intérêt égoïste du mari ou du père. C'est même le dieu Amour qui rendra les amoureux rusés :

Soyez amant, vous serez inventif :

Tour ni détour, ruse ni stratagème

Ne vous faudront : le plus jeune apprentif

Est vieux routier dès le moment qu'il aime. (La Fontaine, «Le Cuvier », p. 875)

\footnotetext{
8 «[Q]uand, dédoublé en son ego fictif et protégé par l'immatérialité de celuici, le lecteur peut s'offrir le plaisir de ruser impunément avec les acteurs de l'illusion romanesque et même de courir le risque de se laisser prendre, innocemment et sans conséquences réelles, dans l'engrenage de la ruse et de son écriture. » (Grodek et Boursier, « Avant-propos », 2000, p. 20).
} 
De ce point de vue, la ruse n'est pas employée gratuitement par les personnages, ni malicieusement, mais comme l'ultime ressource face à une tension dramatique provoquée par des convoitises contradictoires et incompatibles, facilitant ce que Sternberg nomme « la résolution sans éclat des conflits » (p. 3738). Elle répond à des logiques de pouvoir, devient le recours du faible devant le fort qui abuse de son pouvoir, dédoublant une opposition entre la force et l'intelligence ${ }^{9}$. Les femmes et les fils, les servantes et les valets l'emploient contre les pères ou les maris, qui disposent de la force ou de l'autorité. La ruse émerge d'un contexte où les problèmes de droit semblent insolubles, où les intérêts des différents partis culminent en une opposition insoutenable; elle louvoie dans des chemins de traverse qui permettent de s'arroger un droit qui est contesté.

Cet amalgame de l'abus de pouvoir et de l'impuissance réduite à des manœuvres douteuses justifie l'utilisation de la ruse et la situe dans une dynamique qui l'excuse à partir d'une bonne cause : abuser ceux qui nous abusent sera toujours facile à défendre sur le plan légal puisqu'il s'agit d'une action juste. C'est pourquoi la mécanique du trompeur trompé a connu autant de succès dans les deux genres qui nous intéressent, non seulement parce que la dynamique du retour des faveurs crée souvent la surprise agréable et le rétablissement des forces (revanche du faible sur le fort qui a de quoi plaire au parterre), mais surtout parce que l'aspect moral de cette dynamique répond à une remise à niveau, selon laquelle on rétablit la justice en faisant subir une injustice à celui qui vient d'en causer une, d'autant qu'on ressent un plaisir fébrile à voir des faibles

9 Vernet (2000) décrit la ruse comme l’«intelligence au service de la faiblesse », p. 30 . 
se venger après avoir subi de tels dommages. Toutefois, la topique du "trompeur trompé » est plus fréquente dans les contes de La Fontaine ${ }^{10}$ que chez Molière, comme si cette dynamique de l'abus et de la revanche, de la victoire du petit sur le grand, appartenait davantage au registre de la narration et que cette topique convenait moins à la scène du XVII ${ }^{e}$ siècle.

Aux questions de pouvoir se superpose l'esthétique du ridicule étudiée par Patrick Dandrey (2002 [1992]) et centrale dans la dramaturgie de Molière : c'est cette association d'une autorité souffrant d'un ridicule qui assure la plus grande efficacité du comique. Plutôt que d'entraîner un échange circulaire entre un trompeur et un trompé, qui devient trompeur à son tour, il semble que la logique générique des farces et des contes rende certains personnages toujours susceptibles d'être la cible des tromperies et que le personnage ridicule se fasse lui-même l'artisan de ses malheurs, soit par la fausse confiance qu'il a en ses moyens, soit par l'aveuglement que cause sa monomanie, qu'on pense à la folie ou à certains vices comme la jalousie, l'avarice, l'hypocondrie ou la bigoterie. La ruse est doublement justifiée: d'abord parce qu'elle confirme la bêtise des personnages ridicules et amène le public à se divertir à leurs dépens; ensuite parce que ces derniers n'ont que ce qu'ils méritent, ayant eux-mêmes en premier lieu soumis leurs proches à un pouvoir tyrannique pour avancer leurs propres intérêts. Ainsi, les questions éthiques et juridiques à la base du comique servent à atténuer la gravité sociale et morale de l'utilisation de ce recours, à légitimer ce

\footnotetext{
${ }^{10}$ Voir, par exemple, les contes « Le Mari confesseur », « Le Faiseur d'oreilles et le raccommodeur de moules », « Le Gascon puni » et « Les Quiproquos ».
} 
procédé et à le rendre acceptable en le plaçant dans une double logique du pouvoir abusif et d'une esthétique du ridicule.

Toutes ces remarques permettent de confronter la ruse à d'autres notions connexes comme l'erreur, la fourberie ou l'imposture, qui n'ont pas la même portée dans un contexte comique. Si la ruse essaie toujours de mettre un personnage dans l'erreur, celle-ci n'est pas nécessairement preuve de ruse puisqu'elle n'implique pas une intention de tromper, comme on peut voir dans Sganarelle, ou Le cocu imaginaire. L'intrigue de cette pièce progresse grâce à une série d'erreurs de perception, voire de conclusions erronées sur des prémisses douteuses, alors que les deux couples ne songent jamais à être infidèles ${ }^{11}$. Quand la fourberie atteint à la malhonnêteté, elle est parfois dénoncée comme un mal et la comédie se trouve à ce moment «porteuse d'une réflexion éthique» (Sternberg, 1999, p. 37). Les manigances de Béline dans Le Malade imaginaire n'entrent pas dans les cas typiques de la ruse, parce qu'elles sont rattachées à une intention de tromper tournée vers un égoïsme qui entend dépouiller un personnage de ses possessions, ce qui contrevient aux accords implicites du pacte comique. L'hypocrisie de Dorante, dans Le Bourgeois gentilhomme, serait également à classer dans cette catégorie ${ }^{12}$. Ce type de personnage fourbe ne s'attirera jamais la sympathie du public et est susceptible de se voir puni à la fin, au contraire de

\footnotetext{
${ }^{11}$ Le début de l'intrigue pourrait se résumer ainsi : Sganarelle voit sa femme avec le portrait d'un homme dans les mains ; selon lui, ce ne peut être que son amant ; il se croit donc cocu ; or c'est un portrait qu'elle a ramassé dans la rue sans savoir de qui il est. Voir Molière, OEuvres complètes (1971), t. I, p. 299334. C'est l'édition citée dans la suite de l'article.

12 À propos duquel Sternberg affirme : «L'éclairage est porté sur la peinture psychologique du rusé, peinture qui permet cette fois de renvoyer à une réalité [...] peu glorieuse. » (1999, p 37).
} 
certains valets rusés comme Mascarille ${ }^{13}$, Sbrigani ou Scapin. On peut aussi tracer, comme le fait McKenna (p. 29-36), une évolution entre la fourberie et l'imposture, celle-ci ayant une portée satirique ou cynique plus grande, comme on peut le voir avec les figures de Tartuffe et de Dom Juan. L'imposture chez ces deux personnages est fondée sur un décalage entre la dévotion qu'ils projettent ( $\mathrm{du}$ moins pour le Dom Juan du cinquième acte) et la mécréance qu'ils cachent mais qui est peu à peu dévoilée. La réception de ces pièces et la réaction de Sganarelle au dernier acte prouvent bien que ces personnages provoquent plutôt le scandale que le comique franc ${ }^{14}$.

Il faut tout de même préciser que les farces de Molière et les contes de La Fontaine ne sont pas étrangers aux courants hédoniste et libertin propres au milieu du XVIIe siècle ${ }^{15}$. Ces genres diffusent une morale joyeuse fondée depuis leur origine sur un code de comportement gaulois et une échelle de valeurs orientée vers le plaisir. L'amour et l'assouvissement des passions sont présentés comme des fins acceptables, voire louables, et une femme mal mariée sera toujours justifiée de trouver un amant à sa mesure. Le cocufiage et l'adultère sont des thèmes indissociables du genre et l'amour réciproque constitue une motivation plus forte que l'argent, la religion, le statut ou l'occupation. Triomphe aussi une morale de l'accommodement que les casuistes ne manqueraient pas de qualifier de laxiste, que ce soit la discrétion des maris qui se savent cocus, les accommodements de Tartuffe

\footnotetext{
${ }^{13}$ Celui de L'Étourdi, et non celui des Précieuses ridicules.

14 «O Ciel! qu'entends-je ici? Il ne vous manquait plus que d'être hypocrite pour vous achever de tout point, et voilà le comble des abominations » (Dom Juan, $\mathrm{V}, 2$, p. 81). Pour un développement sur la réception de cette pièce, voir la notice de Couton (t. II, p. 3-30).

15 Mes analyses rejoignent à cet égard les travaux de McKenna (2005) et de Darmon (1998).
} 
quant à ses plaisirs secrets, ou les détails gênants que la fiancée du roi de Garbe omet dans le récit qu'elle fait à son mari (La Fontaine, p. 685-686). Le libertinage se remarque même dans des contes qui devraient contenir une finalité morale et pédagogique, des contes d'avertissement à la manière $\mathrm{du}$ « Petit Chaperon rouge », où le but n'est pas de faire rire aux dépens de la dupe, mais bien de causer une réaction négative à l'égard du trompeur et d'informer les lectrices des procédés malhonnêtes, comme dans «L'Ermite » ou dans « Comment l'esprit vient aux filles ». Le fait même d'accuser certains moines ou abbés d'abuser les jeunes filles relève ici d'un anticléricalisme qui passe à cette époque pour une forme du libertinage dans la mesure où il reconduit des accusations quant à l'hypocrisie des religions et l'aspect manipulateur de la notion de péché. La ruse sexuelle préfigure et laisse deviner l'imposture politique fondatrice de toute religion révélée. D’ailleurs, le plaisir sexuel est présenté positivement dans ces deux contes, ou comme un mal nécessaire, ce que prouvent les conséquences profitables qu'en retirent Lisette (elle acquiert de l'esprit) et l'autre personnage anonyme (elle perd ses illusions et son ambition ridicule de devenir la mère d'un pape). C'est donc un soubassement éthique fortement marqué par des postures hétérodoxes, même anti-chrétiennes, qui détermine l'utilisation d'une topique particulière à la ruse dans le contexte comique des contes et des comédies.

\section{Pour une topique de la ruse}

Dans son étude des Contes à rire du Moyen Âge, Philippe Ménard a bien posé la difficulté de catégoriser les types de ruses: «la 
ruse est multiforme, [...] elle se joue des liens dans lesquels nous voulons l'enserrer» (p.26). La variété des termes qui forment le vocabulaire de la ruse en est une preuve assez tangible : «tours », « stratagèmes », «tromperie », «fourberie», « ressorts», « machines », « affaire», « invention», « batterie» sont utilisés par les personnages ou par le narrateur pour décrire ces procédés, de même que plusieurs expressions populaires comme « faire tomber dans le panneau », « jouer une pièce », « en donner » ou, son contraire, « en tenir ». De plus, la répartition des rôles dans la pièce participe à la construction des ruses dans les genres comiques, et les rôles opèrent presque toujours la séparation entre les fourbes et les dupes. Les personnages rusés sont souvent annoncés dès l'exposition de la pièce, comme Mascarille dans L'Étourdi, Sbrigani dans Monsieur de Pourceaugnac ou Scapin dans Les Fourberies. Ces personnages font avancer l'intrigue par leurs ruses, leurs tours et leur adresse. Au théâtre, du moins chez Molière, ils ne sont jamais les héros amoureux principaux, mais des adjuvants qui prêtent leur intelligence et leur savoir-faire aux jeunes premiers. Les dupes entrent aussi dans le personnel nécessaire à la ruse, notamment les pères ou les maris, caractérisés par leur sottise ou par leur propre folie, comme Arnolphe ou le Sganarelle de L'École des maris. La situation des contes est légèrement différente, dans la mesure où le trompeur peut être autant le galant, comme dans "Richard Minutolo», "Le Muletier » ou « La Clochette », que la femme, dans « Le Gascon puni » et « Le Berceau».

Pour narrer la ruse, l'auteur fait face à des exigences complexes à partir du moment où il souhaite ou non inclure le lecteur, l'avertir de ce qu'il verra et le placer dans une posture de juge des bons coups, ou au contraire ménager la surprise, 
l'amener à percevoir progressivement l'action qui se déroule sous ses yeux. Une tension se crée ainsi entre l'action des personnages et leur intentionnalité, tension qui dédouble la temporalité sur le plan narratif, entre l'expectative d'un «regardez-moi bien le tromper » et la surprise de l'explication finale : «il s'agissait d'une feinte». Max Vernet exposait cette spécificité de la narration: "pas de ruse, de ruse thématisée, j'entends, de ruse racontée, sans un récit à double détente qui revienne sur ses pas pour compléter l'information donnée au lecteur. [...] Pas de ruse, au fond, sans analepse » (p. 33). Sans prolepse non plus, devrait-on ajouter, puisque le but de la ruse est souvent énoncé dès la fin de l'exposition ou de l'exorde, comme quand Mercure décrit Jupiter dans le prologue d'Amphitryon :

Ses pratiques, je crois, ne vous sont pas nouvelles :

Bien souvent pour la terre il néglige les cieux;

[...]

Et sait cent tours ingénieux,

Pour mettre à bout les plus cruelles.

Des yeux d'Alcmène il a senti les coups;

Et tandis qu'au milieu des béotiques plaines,

Amphitryon, son époux,

Commande aux troupes thébaines,

Il a pris la forme, et reçoit là-dessous

Un soulagement à ses peines

Dans la possession des plaisirs les plus doux. (Molière, 1971, t. II, p. 363)

Ces récits sont quelquefois accompagnés d'une ellipse qui construit une forte attente chez le spectateur, effet susceptible de ménager la surprise, comme dans cette première scène des Précieuses ridicules :

La Grange : [...] Je vois ce qu'il faut être pour en être bien reçu; et si vous m'en croyez, nous leur jouerons tous deux une pièce 
qui leur fera voir leur sottise, et pourra leur apprendre à connaître un peu mieux leur monde.

Du Croisy : Et comment encore?

La Grange : J'ai un certain valet, nommé Mascarille, qui passe, au sentiment de beaucoup de gens, pour une manière de bel esprit [...].

Du Croisy : Eh bien, qu'en prétendez-vous faire?

La Grange: Ce que j'en prétends faire? Il faut... Mais sortons d'ici auparavant. (Molière, 1971, t. I, p. 266)

Le spectateur ne saura pas avant la septième scène la suite de cette ruse, qui attrapera les deux précieuses et sera dévoilée au dénouement avec le retour des deux hommes.

Dans le cas des contes, ces procédés narratifs sont actualisés par différents outils, notamment dans "Le Muletier», où l'utilisation du passé simple donne au récit une orientation vers le futur, autant dans les vers du début:

Pour se guérir [de sa passion pour la reine], sans pouvoir rien gagner,

Le compagnon fit un tour d'homme habile (La Fontaine, p. 624). que dans ceux de la fin :

[...] le galant s'avisa d'un secret

Qui d'Agiluf gâta le stratagème. (p. 627)

Ces formules ont pour fonction d'annoncer ce qui va suivre; d'ailleurs, le verbe «s'aviser» revient fréquemment dans les contes pour donner au lecteur un avant-goût des événements qu'il va bientôt lire. Le procédé inverse se trouve dans « On ne s'avise jamais de tout », après que la femme d'un jaloux eut reçu un panier d'ordures sur la tête, ce qui met la puce à l'oreille à ce dernier :

[...] Foin, dit-il, celui-là [ce procédé]

N'est pas dans mon livre, et je suis pris pour dupe;

Que le recueil au diable soit donné! (p. 659-660) 
Le narrateur vient alors prendre le relais de la narration après la réaction du cocu jaloux, en expliquant l'intentionnalité derrière ce geste urbain habituellement burlesque, mais qui acquiert ainsi le statut de ruse par sa finalité :

Il disait bien; car on n'avait jeté

Cette immondice, et la dame gâté,

Qu'afin qu'elle eût quelque valable excuse

Pour éloigner son dragon [la servante] quelque temps. (p. 660)

Qu'elle soit narrative ou dramatique, la ruse implique divers jeu entre la temporalité de l'action et celle de la narration, crée des effets d'annonce qui sont suspendus, raconte sans tout expliquer, ou le fait avec un temps de retard, suscitant ainsi l'attente et l'indécision, toujours en piquant la curiosité du lecteur ou du spectateur et en jouant sur des effets de surprise.

À cet égard, puisque l'appréciation de la ruse et l'observation de son déroulement forment une grande partie du plaisir du lecteur, Molière et La Fontaine se permettent souvent des appels directs au public ou trouvent parfois des moyens métalittéraires de faire comprendre la ruse à l'auditoire. Le langage technique et littéraire vient alors se greffer sur les dialogues des personnages, comme on le remarque à la présence de termes ou d'expressions comme "personnages", "faire une pièce », " apprêter à rire », ou encore dans ces deux répliques de Monsieur de Pourceaugnac:

Sbrigani : [...] laissons cela; et pour commencer notre affaire, nous allons vite joindre notre provincial, tandis que, de votre côté, vous nous tiendrez prêts au besoin les autres acteurs de la comédie.

Éraste: Au moins, Madame, souvenez-vous de votre rôle; et pour mieux couvrir notre jeu, feignez, comme on vous a dit, d'être la plus contente du monde des résolutions de votre père. (I, 3, Molière, t. II, p. 596) 
Certes, les monologues des rusés et les dialogues des amants avec leurs confidents marquent toujours la transparence de leurs intentions, mais d'autres stratagèmes sont souvent mis en œuvre, comme les apartés ou les aveux du narrateur ${ }^{16}$. Quand Mascarille termine son monologue par «Bon, voyons si son feu se rend opiniâtre » (L'Étourdi, III, 1, Molière, t. I, p. 98), cet impératif à la première personne implique le spectateur dans les desseins du personnage, créant une complicité entre le rusé et son observateur. La ruse dépasse ainsi l'intrigue entre les personnages et occasionne une rupture du mur invisible entre la salle et la scène, mesures prises pour que le lecteur ou le spectateur ne soit pas victime de la ruse mais puisse l'observer et mieux la goûter ${ }^{17}$.

La ruse elle-même se sert de nombreux expédients qui montrent le caractère indissociable de la parole et de l'action. Dans les procédés qui emploient surtout la parole, on peut évoquer le mensonge, la flatterie, le faux serment ou la fausse promesse, les aveux dissimulés, la double entente qui appelle la méprise ${ }^{18}$. Pour ne donner qu'un exemple de mensonge, le personnage principal de «Richard Minutolo» monte une calomnie auprès de la dame qu'il veut séduire, en prétendant que son mari donne rendez-vous à sa maîtresse dans une chambre sombre. La femme s'y rend, croyant attraper son mari, mais elle est bien surprise quand elle découvre que Minutolo lui-même est dans le lit (La Fontaine, p. 572-577). Quant aux

\footnotetext{
${ }^{16}$ Voir notamment ces vers du conte « Le Petit chien qui secoue de l'argent et des pierreries »: «Pour le More lippu [qui voulait forcer l'époux], c'était Manto la fée, / Par son art métamorphosée, / Et par son art ayant bâti / Ce Louvre en un moment [...]. » (La Fontaine, p. 775)

${ }^{17}$ Sternberg affirme à ce propos : « Ces comédies d'intrigue fondent en effet le plaisir du spectateur sur la contemplation du jeu. » (p. 37)

${ }^{18}$ C'est le cas des contes « Le Mari confesseur » et « Le Berceau ».
} 
aveux dissimulés, ils se trouvent dans L'École des maris et dans "La Confidente sans le savoir», où l'amante se sert d'une personne interposée pour porter ce qui ressemble à une plainte au premier abord, mais qui, interprétée correctement par son destinataire, c'est-à-dire à rebours, contient un véritable message d'amour. Ce premier message mène à des échanges de lettres ou de présents et, finalement, à un rendez-vous pris en l'absence du mari ou du père intéressé. Pour les moyens non verbaux et qui fabriquent une ruse d'action, l'on trouve le plus souvent le déguisement, la simulation de ce qui n'est pas (Mazet de Lamporechio feint d'être muet et stupide), la cachette (notamment du mari pendant qu'on séduit sa femme, dans Tartuffe et dans «Les Rémois »), le quiproquo, enfin la magie dans Amphitryon et dans certains contes comme «La Coupe enchantée » et «Le Petit chien qui secoue de l'argent et des pierreries ${ }^{19}$.

La vérité controuvée mérite un commentaire séparé : les amants avoueront ouvertement leur intention au mari ou au père, sans que cet aveu soit pris pour une vérité. Ce procédé se trouve dans L'Amour médecin et dans «Le Cocu battu et content». Dans ce conte, la femme avoue à son mari que leur nouveau valet lui a parlé d'amour, mais elle lui donne une fausse information quant au lieu du rendez-vous. Le soir venu, le mari se travestit et va attendre le valet dans le jardin, tandis que les amants s'ébattent à l'intérieur. Le valet se rend ensuite dans le jardin pour gronder la femme ; il feint de lui avoir donné un rendez-vous afin de tester sa fidélité : comme elle se déclare déloyale en s'y trouvant, elle mérite d'être battue. Le mari,

\footnotetext{
${ }^{19}$ On peut également se référer au chapitre de Catherine Grisé sur la magie et l'illusion, p. 65-105.
} 
amené à croire que son valet est vraiment trompé par le déguisement et qu'il le prend pour sa femme, sera content d'être battu, puisque c'est une preuve que sa femme et son valet lui sont tous deux loyaux. Il ignorait en fait que son valet n'était à l'origine qu'un galant déguisé, et non un valet de ferme. Un tour semblable est joué à Sganarelle dans L'Amour médecin quand Clitandre, l'amant de sa fille, se déguise en apothicaire et prétend que tromper sa fille en lui parlant de mariage la guérira de sa folie. Aveux réciproques, demande en mariage officielle et signature du contrat notarié peuvent donc s'effectuer sous les yeux du père, jeu d'autant plus drôle que ce dernier croit sa fille folle et pense que c'est elle qui est jouée. Il s'aperçoit qu'il est la seule dupe à la fin de la pièce et que ce n'était pas une feinte. Le mécanisme de la vérité controuvée implique ainsi un déguisement qui trompe l'un des personnages, mais dont le public est averti préalablement, ce qui occasionne un double jeu d'interprétation entre le mari cocu (ou le père) et le spectateur qui voit la ruse s'accomplir sous ses yeux.

Même si la ruse est un moteur privilégié de l'intrigue, elle n'est pas pour autant en lien direct avec le dénouement. En effet, le coup de théâtre ou la mécanique du deus ex machina expliquent la fin de plusieurs pièces, qui montrent l'inanité finale de la ruse, comme dans Les Fourberies de Scapin ou dans L'Étourdi, où l'apparition d'un père et les reconnaissances finales viennent dénouer les difficultés qui empêchaient la réussite des mariages. La ruse ne serait donc pas toujours une cause directe de la fin prévue par les personnages, mais un mécanisme de remplissage entre une situation initiale embrouillée et une situation finale attendue. Elle deviendrait alors un topos générique fondateur du plaisir théâtral ou littéraire, de la même manière que le combat ou le voyage 
fantastique forme la trame d'une narration épique. En tant qu'«incident comique par excellence» (Guardia, p. 32) qui suscite un rire de moquerie, la ruse stimule la création de situations loufoques et constitue un facteur de liaison entre les principaux moments comiques. Le rapport entre la ruse et le dénouement montre ainsi une tendance forte de la farce et de la comédie, selon laquelle l'intrigue serait secondaire et l'unité scénique serait tributaire des procédés de ruse et de tromperie, alors que dans les contes, l'un des plaisirs du dénouement est de savoir si la ruse a réussi ou non, ce qui donne parfois lieu à un bon mot ou à une courte leçon.

\section{Les écarts de la logique}

Il semble en dernier lieu que la ruse soit indissociable non seulement $d u$ mensonge, mais aussi des détours de l'argumentation, comme si la combinaison des prémisses et des conclusions constituait un facteur essentiel des réactions des personnages. D'ailleurs, La Fontaine montre bien son appréciation des codes logiques au début de «Mazet de Lamporechio ", quand il dénonce les erreurs des parents qui mettent leur fille au couvent :

Ma fille est nonne, ergo c'est une sainte, Mal raisonner. (p. 693)

On sait que la logique et la rhétorique, avec laquelle elle partage des origines communes, sont toutes dévouées à faire entrer les locuteurs en accord, voire à manipuler l'opinion et la pensée afin d'orienter le choix des actions (voir Arnauld et Nicole, 1992, et Declercq, 1992). Puisque le comique est souvent une 
question d'intelligence (ou, selon Bowen, p. 29-31, de sottise), de distance et de supériorité, différents types d'erreurs de logique placent le public dans une posture favorable au rire et conditionnent son rapport avec les personnages. Par leurs erreurs de raisonnement et certains abus dans l'argumentation, les personnages donnent un signe évident qu'ils sont les ridicules et prêtent le flanc à la moquerie. Une dichotomie peut alors se créer dans l'esprit du spectateur entre les ridicules et les raisonneurs, qui doivent attirer des réactions différentes pour que fonctionne le spectacle ${ }^{20}$. Des erreurs présentes dans les contes servent quelquefois à ajouter un enjouement plaisant dans le texte ou à lancer une intrigue. Un premier type d'erreurs consiste à opérer des analogies absurdes, comme dans «Pâté d'anguille »21, où la satiété d'un mets et la nécessité de varier les plaisirs de la bouche vont servir à prouver l'importance de l'infidélité et du libertinage. Dans le cas des « Troqueurs » (La Fontaine, p. 819-823), une question fondée sur une analogie semblable va lancer les personnages dans une aventure plaisante par sa naïveté. Ils se demandent: si deux personnes peuvent échanger leur cheval, pourquoi ne pourraient-ils pas aussi échanger leur femme? Le plaisant de ces deux analogies est contenu dans le décalage abusif - et burlesquement dépréciatif - entre la femme, la nourriture et les chevaux.

Plusieurs sophismes entrent aussi dans la logique erronée des contes, le plus fréquent étant le pouvoir argumentatif de l'argent, qui réussit à inciter les belles à

\footnotetext{
${ }^{20}$ Sur la question des raisonneurs dans le théâtre de Molière, se rapporter à Hawcroft (2007).

${ }^{21}$ C'est le conte qui contient le fameux vers «Diversité c'est ma devise » (La Fontaine, p. 863-866).
} 
l'infidélité, comme dans «À femme avare galant escroc » (La Fontaine, p. 657-658). Dans «L'Hermite», ce sera un abus d'autorité qui perdra la belle, qui croit entendre la parole directe de Dieu et se plie à ses volontés, croyant faire une action louable en allant voir le frère Luce (p.687-692). Les jeux entourant l'équivoque formeront un autre type d'abus de logique, où les mots et leurs sens seront amenés à permuter entre eux, encore au détriment de quelques belles. Dans «Comment l'esprit vient aux filles", le père Bonaventure répond à la requête de la jeune Lise, qui vient dans sa cellule pour chercher de l'esprit, ce que le bon père refuse de lui vendre, mais lui donne de bon gré à plusieurs reprises après l'avoir jetée sur le lit (p. 811-814). Le cas de frère Rustic et de la demoiselle Alibech est similaire dans «Le Diable en enfer »: cette dernière souhaitait acquérir de la sainteté auprès d'un ermite, mais elle est bien trompée par un nouveau " mystère » agréable à Dieu et qu'elle ne connaissait pas, où il s'agit littéralement d'emprisonner «en enfer le Malin» (p. 855). Si ces équivoques servent à tromper les jeunes filles dans le récit, elles permettent au narrateur d'aborder les choses de l'amour avec une plus grande liberté, décuplant le plaisir du lecteur amusé par la virtuosité du code utilisé, que ce soit le nombre de «doses d'esprit » données à Lise et la « charité » du bon père Bonaventure, ou la capacité de la vierge Alibech d'accoutumer sa "prison » à accueillir le «diable » et rendre sa "présence agréable » (p. 855-856).

Il faut aussi noter toutes les fautes de conclusion qui sont amenées par des prémisses plus ou moins vraies, comme celles déjà évoquées de Sganarelle dans Le Cocu imaginaire, où le portrait d'homme dans les mains de sa femme est pris pour celui de son galant, ou quand Sganarelle tient une femme dans 
ses bras, elle semble être son amante, et non une femme évanouie qu'on aide à remettre sur pied. Pareille erreur d'interprétation se retrouve dans "La Clochette», au moment où la jeune fille cherche sa vache perdue en tentant de suivre le son de sa clochette, le son étant ici pris pour l'indice de la présence effective de la vache. Elle sera bien surprise de constater qu'un galant tenait cette clochette justement pour l'attirer au fond des bois: sa mauvaise conclusion sur des signes ambigus la rend victime de la ruse de son amant. L'erreur d'Agnès dans L'École des femmes est plus plaisante parce que naïve. Quand Arnolphe lui explique que le mariage enlève le "crime» en amour, elle conclut qu'en épousant ce dernier, elle pourra prendre du plaisir avec son amant Horace :

Hélas! que je vous ai grande obligation,

Et qu'avec lui j'aurai de satisfaction! (II, 5, Molière, t. I, p. 577)

Tout cet imbroglio aurait pu être évité en clarifiant les pronoms rattachés au verbe «se marier » et en élucidant l'ambiguïté qui persiste entre les expressions "vous donner en mariage » ou "vous épouser moi-même », mais c'est ici la grande habilité du dramaturge qui permet de rendre cette faute de logique amusante et vraisemblable.

Les abus de logique apparaissent également pour dénoncer l'incompétence des médecins, notamment dans Monsieur de Pourceaugnac. Les deux médecins sont avertis $a$ priori que ce dernier est fou, ce que leur interrogation corporelle ne fera que confirmer. Leur diagnostic et la définition de la mélancolie hypocondriaque appellent toutes les autorités habituelles, comme Esculape, Hippocrate et Galien, et ils accomplissent les examens du corps orientés par la théorie des humeurs: par exemple, le fait de bien manger est une 
« indication de la chaleur et sécheresse qui est au-dedans » (I, 8, Molière, t. II, p. 607). Ils formulent des liens ad absurdum qui confondent la cause avec la conclusion, où «la maladie dont, par notre raisonnement, il est manifestement atteint et convaincu » (p.608), comme si le raisonnement était la cause de la folie, erreur confirmée par le second médecin :

Vous avez si bien discouru sur tous les signes, les symptômes et les causes de la maladie de Monsieur; le raisonnement que vous en avez fait est si docte et si beau, qu'il est impossible qu'il ne soit pas fou, et mélancolique hypocondriaque; et quand il ne le serait pas, il faudrait qu'il le devînt, pour la beauté des choses que vous avez dites, et la justesse du raisonnement que vous avez fait. (p. 610)

Les médecins sont encore moqués dans Le Médecin malgré lui, où il $\mathrm{y}$ a une double feinte: Sganarelle n'est pas médecin et Lucinde n'est pas malade. Ce faux médecin commence par définir le problème de manière tautologique, en affirmant que la fille est muette " parce qu'elle a perdu la parole », à cause de «l'empêchement de l'action de la langue » (II, 4, Molière, t. II, p. 245). Il commet ensuite de nouvelles bourdes en évoquant Aristote mais en ne le citant pas (qui « dit... De fort belles choses »), il définit par redondance «humeurs peccantes; peccantes, c'est-à-dire... humeurs peccantes »; il prend enfin les règles de grammaire de Despautère pour des aphorismes de médecine, pour la seule raison qu'elles sont en latin et qu'elles sont apprises à l'école, ce qui montre qu'il n'a pas assimilé les rudiments de l'éducation. Son analyse devient plus précise quand il se met à expliquer la pathologie par l'influence des vapeurs noires parmi les organes du corps, mais il se trompe grossièrement dans la physionomie (il met le cœur à droite et le foie à gauche). Et comme si le raisonnement de Sganarelle n'était pas assez comique en lui-même, Molière ajoute une 
conclusion ouvertement stupide par Géronte : «On ne peut pas mieux raisonner, sans doute» (p. 246), ce qui conforte le spectateur dans sa position d'intelligence et de supériorité, qui perçoit l'ironie d'une telle formule et qui voit bien qu'au contraire, il est plus que possible de « mieux raisonner ».

De même, pour dénoncer les abus de l'hypocrisie religieuse, Molière montre à quel point les arguments de Tartuffe sont fuyants et réversibles ${ }^{22}$. Devant Elmire, il est homme, et la désirer est un effet de la grandeur divine (III, 3). Une fois confronté, il avoue qu'on peut s'accommoder avec le Ciel sans l'offenser en dirigeant l'intention (IV, 5, t. I, p. 963). Il est cependant beaucoup plus ferme avec Cléanthe au début de l'acte IV quant aux « intérêts du Ciel » (p. 953), puisque « le Ciel l'ordonne» (p. 954) et qu'il travaille pour la gloire du Ciel. De tels glissements casuistiques se retrouvent aussi chez La Fontaine, dans "Les Frères de Catalogne » (p. 612-618), où la notion de dîme est appliquée aux « œuvres du mariage », ce que les moines sont prêts à faire pour que les dames du lieu aillent directement au paradis, malgré la peine que cela leur coûtera et qu'ils prendront «en patience» (p. 614), le tout autorisé par les écritures, le pape et Dieu lui-même. On retrouve ici encore les interactions entre la ruse littéraire et l'imposture religieuse, où la présence de la ruse dans les textes suggère au lecteur averti des pistes de réflexion sur les mœurs, l'argumentation et les désagréments dans lesquels tombent les victimes de tels abus.

Ce regard croisé sur les comédies et les contes montre que ces genres vont souvent par paires quand il s'agit de la ruse

${ }^{22}$ Sur Tartuffe, voir McKenna, p. 37-43. Grisé (p. 161-1802010) a également rédigé un chapitre sur la casuistique dans les contes. 
et que les mêmes mécanismes fonctionnent de manière similaire, ce qui confirme à quel point ils possèdent des frontières communes quant à l'éthique, la topique et la logique. Mais en déployant une caricature de la rhétorique des religieux dans leurs œuvres, Molière et La Fontaine participent ultimement à la dévalorisation par le comique de la posture de l'Église, accusée d'abuser de la confiance des fidèles, à laquelle on applique une logique du dévoilement, de mise à bas des masques qui permet de dénoncer l'imposture, la ruse dont les fidèles sont les dupes. L'étude de la ruse dans le conte et la comédie montre l'importance des finalités didactiques de ces genres et illustre l'appartenance de ces deux auteurs au libertinage et à l'épicurisme du XVIIe siècle.

\section{Bibliographie}

ARnAUld, Antoine, et Pierre Nicole. (1992), La Logique ou l'art de penser, Paris, Gallimard.

BÉnichou, Paul. (1948), Morales du grand siècle, Paris, Gallimard.

BoWEN, Barbara. (1964), Les Caractéristiques essentielles de la farce française et leur survivance dans les années 1550-1620, Urbana, University of Illinois Press.

Collinet, Jean-Pierre. (1970), Le Monde littéraire de La Fontaine, Paris, PUF. 
DANDREY, Patrick. (1998), La Médecine et la maladie dans le théâtre de Molière, Paris, Librairie Klincksieck, 2 vol.

—. (2002 [1992]), Molière ou l'esthétique du ridicule, Paris, Librairie Klincksieck.

DARMON, Jean-Charles. (1998), Philosophie épicurienne et littérature au XVIIe siècle: Études sur Gassendi, Cyrano de Bergerac, La Fontaine, Saint-Évremond, Paris, PUF, coll. «Perspectives littéraires ».

Dictionnaire de l'Académie française, Paris, J.-B. Coignard, 1694.

DECLERCQ, Gilles. (1992), L'Art d'argumenter: Structures rhétoriques et littéraires, Paris, Éditions Universitaires.

Gilot, Michel, et Jean Serroy. (1997), La Comédie à l'âge classique, Paris, Belin.

Godenne, René. (1995), La Nouvelle, Paris, Honoré Champion.

GRISÉ, Catherine. (2010), Jean de La Fontaine: Tromperies et illusions, Tübingen, Narr Verlag, coll. « Biblio 17 ».

GRODEK, Elzbieta (éd.). (2000), Écriture de la ruse, Amsterdam et Atlanta, Rodopi.

Guardia, Jean de. (2007), Poétique de Molière: Comédie et répétition, Genève, Droz.

HAWCROFT, Michael. (2007), Molière Reasoning with Fools, Oxford University Press.

IMBS, Paul (dir.). (1971-1994), Trésor de la langue française: dictionnaire de la langue du XIXe et du XXe siècle 1789-1960, Paris, Éditions du CNRS. 
LA FontAine, Jean de. (1991), CEuvres complètes I : Fables, contes et nouvelles, éd. Jean-Pierre Collinet, Paris, Gallimard, coll. «Bibliothèque de la Pléiade ».

LEWICKA, Halina. (1974), Études sur l'ancienne farce française, Paris, Klincksieck.

McKenna, Antony. (2005), Molière dramaturge libertin, Paris, Honoré Champion, coll. «Champion Classiques ».

MÉnARD, Philippe. (1983), Les Fabliaux : contes à rire du Moyen Âge, Paris, PUF.

Molière, Jean-Baptiste Poquelin, dit. (1971), QEuvres complètes, éd. Georges Couton, Paris, Gallimard, coll. « Bibliothèque de la Pléiade », 2 vol.

REY-FLAUD, Bernadette. (1996), Molière et la farce, Genève, Droz.

SoulLLER, Didier. (2004), La Nouvelle en Europe : de Boccace à Sade, Paris, PUF.

Sternberg, Véronique. (1999), La Poétique de la comédie, Paris, Sedes.

VERnET, Max. (2000), "Narrer (-) la ruse », dans Elzbieta Grodek (éd.), Écriture de la ruse, Amsterdam et Atlanta, Rodopi, p. 23-36.

VolTz, Pierre. (1964), La Comédie, Paris, Armand Colin. 


\title{
Résumé
}

Cet article propose une analyse de la ruse dans les farces de Molière et dans les contes de La Fontaine afin de mesurer les rapprochements et les différences entre ces deux genres. L'étude porte en premier lieu sur les enjeux éthiques et juridiques, permettant à la fois de susciter le rire auprès du public, tout en dénonçant le ridicule du personnage de la dupe, pour ensuite procéder à l'établissement d'une liste des principaux lieux communs sur lesquels se fonde cette mécanique du rire. L'article se termine sur des considérations logiques et argumentatives associées au comique de la ruse, montrant ainsi comment ces deux genres dévoilent leur dette à l'égard du climat libertin et libre-penseur du XVIIe siècle.

\begin{abstract}
This article provides an analysis of deception in the farces of Molière and La Fontaine's contes to measure parallels and differences between these two genres. The study focuses primarily on ethical and judicial issues that can generate the comic, while conveying the dupe character's ridiculous persona, then proceeds to list the main commonplaces underlying these mechanics of laughter. The paper concludes with considerations associated with the logical and argumentative comic of ruse, showing how these two genres pertain to the climate of libertine and freethinking of the seventeenth century.
\end{abstract}

